\title{
Inhalt des neun und zwanzigsten Bandes.
}

\author{
E r s t e s $H$ e f t.
}

1. ர் ber die Strafsenverbindung der beiden Meere bei Panama. . . . . Seite 1

2. Üler die Aufhebung der Ungleichmäfsigkeil der durch die Kurbel vermittelter Bewegung. Von Herrn Amtmann Prelen zu Ratzeburg. . . . . . - 50

3. Zisätze zu der Abhandlung des Herausgebers No. 2, 10, 13 und 17 im 22ten Bande des Journals für die Baukunst „Über die verschiedenen Arten, ${ }_{n}$ d:e Spannkraft der atmosphärischen Luft als bewegende Kraft auf Eisen„bahnen zu benutzen.”. . . . . . . . . . . . . . . . . . - 53

4. Über Leuchtthürme. Nach englischen, französischen und deutschen Quellen bearbeitet von Herrn Ingenieur $\boldsymbol{A}$. Hefs zu Göttingen. . . . . . . . - 70

$$
\text { Z w e i t e s H e f } t \text {. }
$$

5. Über Leuchtthürme. Nach englischen, französischen und deutschen Quellen bearbeitet von Herrn Ingenieur $\boldsymbol{A}$. Hefs zu Göltingen. (Fortsetzung der Abhandlung No. 4. im vorigen Hefte.) . . . . . . . . . . . . -93

6. Von den Gesetzen der Bewegung der Luft (der Aërodynamik). Nach D'Aubuisson de Voisins. (Schlufs der Abhandlung No. 13. im vorigen Bande.) - 142

$$
\text { D rit t e s H e f t. }
$$

7. Über Leuchtthürme. Nach englischen, französischen und deutschen Quellen bearbeitet von Herrn Ingenieur $\boldsymbol{A}$. $\boldsymbol{H} e / s$ zu Göttingen. (Fortsetzung der Abhandlung No. 4. im ersten und No. 5. im zweiten Hefte dieses Bandes.)

8. Über die italienische Bauart zur Zeit der Wiedergeburt der Künste (Renaissance). Von dem Herrn Oberbaumeister Engclhard zu Cassel in Hessen. 
9. Über die Form der Spitzen einzurammender Pfähle. Von dem Herrn Wegebaumeister Kossak zu Lauenburg in Pommern.

10. Zur Theorie der Gewölbe. Von dem Herrn Bau-Conducteur Scheffler zu Braunschweig. . . . . . . . . . . . . . . . . . . . . . -255

11. Fragen über Fuhrwerkräder. Von Herrn B. auf der Insel Rügen. . . . - 282

$$
\text { Vi e r t e s Heft. }
$$

12. Beschreibung der Bergung des Wracks vom Linienschiffe Christian VIII. Ausgeführt 1849 von Herrn Gullunn, Wasserbau-Inspector zu Hamburg. - 283

13. Zur Theorie der Gewölbe. Von dem Herrn Bau-Conducteur Scheffler zu Braunschweig. (Fortsetzung der Abhandlung No.10. in diesem Bande.) . - 325

14. Über Leuchtthürme. Nach englischen, französischen und deutschen Quellen bearbeitet von Herrn Ingenieur $\boldsymbol{A}$. $\mathrm{He} / \boldsymbol{s}$ zu Göttingen. (Fortsetzung der Abhandlung No. 4. im ersten, No. 5. im zweiten und No. 7. im dritten Hefte dieses Bandes.) . . . . . . . . . . . . . . . . . . . . -349 


\section{J o u r n a l}

f ü r

\section{d i e B a uk u n s t. \\ I $n$ \% w a $n$ g l o s e $n$ I e f t e}

Herausgegeben

vo 11

\section{Dr. A. L. Crelle,}

Königlich-Preufsischem Geheimen-Ober-Banrathe a. D., Rilter des rothen Adler Ordens dritter Classe mit der Schleife, Mitgliede der Königlichen Akademie der Wissenschaften zu Berlin, Correspondenten der Kaiserlichell Akademie der Wissenschaflen zı St. Petersburg und der küniglichen Akademieen der Wissenschaften zn Neapel und Brüssel, auswärtigem Mitgliede der Königlichen Akademie der Wissenschaften zu Stockholm, Elıren-

mitgliede der Hamburger Gesellschaf zur Verbreitung der malhematischen Wissenschaften.

\section{Neun und zwanzigster Band.}

Erstes Heft.

Mitzwei Figurentafelu.

B e r li n.

B. i G. R e i m e r.

1850. 
